\title{
Universal scaling of the magnetic anisotropy in two-dimensional rare-earth layers
}

\author{
L. Benito ${ }^{1,2, *}$ and R. C. C. Ward ${ }^{3}$ \\ ${ }^{1}$ Cavendish Laboratory, University of Cambridge, J. J. Thomson Avenue, Cambridge CB3 OHE, United Kingdom \\ ${ }^{2}$ Departamento de Física de la Materia Condensada, Universidad de Zaragoza, 50009 Zaragoza, Spain \\ ${ }^{3}$ Oxford Physics, Clarendon Laboratory, University of Oxford, Parks Road, Oxford OX1 3PU, United Kingdom
}

(Received 3 February 2015; published 28 July 2015)

\begin{abstract}
Unraveling the influence that low dimensionality has upon the spin's stability in two-dimensional (2D) systems is instrumental for the efficient engineering of energy barriers in ultrathin magnetic layers. Taking rare-earth-based ultrathin multilayered nanostructures as a model system, we have investigated the dissimilar impact that low dimensionality and finite-size effects have upon the magnetic anisotropy energy (MAE) at the nanoscale. We conclusively show that the reduced dimensionality of the spin's system in 2D ferromagnetic layers imprints on the MAE constants a universal temperature decay as a quadratic power law of the reduced magnetization. This result is in agreement with predictions, although in marked contrast to the rank-dependent, thereby faster, decay of the MAE constants observed in three-dimensional nanostructures.
\end{abstract}

PACS number(s): 75.30.Gw, 75.50.Cc, 75.70.Cn

The reliability of spintronics devices [1] crucially resides upon preserving a finely engineered spin structure [2] by optimizing energy barriers [3], which ensures the spin stability and, ultimately, influences decisively device performance [4]. It is thereby unsurprising that the magnetic anisotropy energy [5] (MAE), which determines the magnetic moment orientation [6], defines coercivity and magnetic domain patterns [7], and plays an essential part in spin dynamics [8], has gathered huge interest [9,10], being in the spotlight of countless studies aiming to explore and gain control over the MAE of nanometersized matter [11].

The relentless trend for miniaturization imposes the shrinking of the device size down to the nanoscale, which entails the arising of dominating surface/interface [12] contributions into MAE. In this way, the symmetry breaking of the lattice potential at the nanosystem boundaries gives rise to huge energy barriers $[13,14]$, which are predominantly contributed by perimeter-edge ions [15]. On the contrary, as layer thickness becomes comparable to the spin-spin exchange range, the magnetic ordering temperatures [16-18] are shifted towards lower ones and the spin polarization [19] diminishes faster than in bulk systems as temperature increases. These two facts reflect in the enhancing efficacy of thermally activated spin waves [20] to annihilate long-range magnetic order [21] at the nanoscale.

Newly engineered magnetic two-dimensional (2D) hybrid systems [22] have opened up an exciting new route for faster, more power-efficient spintronics [23], albeit they are not exempt from fundamental challenges. Thus, the reduced dimensionality of the crystal field in transition-metal-based nanosystems is well known to revive orbital moments, which contributes to stabilize the magnetic order in nanometer-sized matter [13,24]. However, the impact that the spin's low dimensionality has upon their thermal stability and, therefore, its crucial role in determining their magnetic response, is still poorly understood. The thermal stability of 2D magnets could be elucidated by measuring the temperature scaling of the model-independent MAE in ultrathin layers; nevertheless, such an experimental test has remained elusive upon until now.

*luisbenito.phys@gmail.com
The Callen and Callen (CC) theory [25] provides a suitable framework within which to analytically model the temperature dependence of the MAE by making use of a single parameter, the reduced magnetization $m$, through which the annihilation of long-range magnetic order is modeled by means of introducing thermally induced spin-wave (SW) excitations. The CC theory has proven immensely successful in a diverse variety of materials, ranging from complex oxides [26], L1-type FePt alloys [27], up to magnetic semiconductors [28], in addition to rare-earth (RE) metals [29-31], forming part of the highly demanded alloys [32].

Here we report on the distinctive way in which finite-size and the spin's dimensionality effects determine the MAE in ultrathin layers. Taking RE superlattices (SLs) as a model system [33], which is featured by the precise modeling of their MAE [29,30], we demonstrate that the two dimensionality of the spin system in ultrathin RE layers imprints a universal, rank-independent temperature scaling on the MAE constant as a quadratic power law of the reduced magnetization, in agreement with early predictions [34].

We have investigated two ultrathin strain-alike RE-based SLs, mainly, $\left[\mathrm{Dy}_{8 \pm 1} / \mathrm{Sc}_{8 \pm 1}\right]_{50}$ and $\left[\mathrm{Ho}_{8 \pm 1} / \mathrm{Lu}_{18 \pm 2}\right]_{75}$, hereafter referred to as Dy/Sc and Ho/Lu SLs, where the subindexes indicate the number of monolayers (MLs) in each layer, and 50 and 75 refer to the number of repetitions of the Dy/Sc and $\mathrm{Ho} / \mathrm{Lu}$ bilayers, respectively. Both SLs were grown by a molecular beam epitaxy technique in a Balzers UMS630 facility, with a base pressure of better than $2 \times 10^{-10} \mathrm{mbar}$ and deposited onto epi-polished (1120)-oriented $\mathrm{Al}_{2} \mathrm{O}_{3}$ substrates, following well-established growth techniques [35,36]. This procedure ensures that the Dy, Sc, Ho, and Lu metallic species, which all crystallize in the hexagonal-closed-packed (hcp) structure [30], grow with the c direction of the hcp lattice structure normal to the deposition plane [35,36], forming high-quality single crystals $[37,38]$.

Magnetic torque measurements were carried out in a vector vibrating sample magnetometer [39]. Data recorded by this apparatus have significantly contributed to a better understanding of the influence that strain-induced magnetoelastic (MEL) terms $[29,38,40]$ have upon the MAE in nanosystems and to demonstrate the anisotropy of the magnetization 

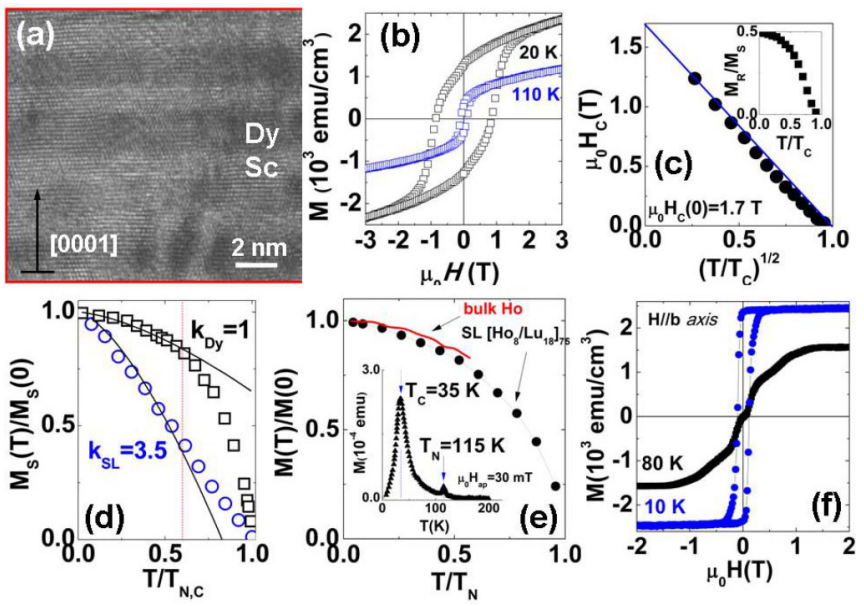

FIG. 1. (Color online) For the $\mathrm{Dy}_{8} / \mathrm{Sc}_{8}$ superlattice (SL): (a) A high-resolution transmission electron microscopy image [38], where dark and light contrast corresponds to the Dy and Sc layers, respectively. (b) Hysteresis loops $M-H$, where the applied magnetic field $\mathbf{H}$ is along the $a$ axis. (c) Coercive field $H_{c}$ vs $t^{1 / 2}$. The line is a fit of the experimental data according to the relationship $H_{c}=$ $H_{c}(0)\left(1-t^{1 / 2}\right)$ [43], where $t=T / T_{C, N}$ is the reduced temperature and $T_{C, N}$ is the Curie or Neél ordering temperature, as appropriate. The graph in the inset shows the ratio between the remanence and saturation magnetization $M_{r} / M_{s}$ vs $t$. (d) Reduced spontaneous magnetization $m_{s}=M_{s} / M_{s}(0)$ vs $t$ for bulk Dy (squares) and the $\mathrm{Dy} / \mathrm{Sc} \mathrm{SL}$ (circles). The lines correspond to a fit of the experimental data according to a pseudo Bloch- $T^{3 / 2}$ law, $m_{s}=\left(1-k_{s} \mathcal{C} t^{3 / 2}\right)$ [49], where $k_{\mathrm{SL}}$ and $k_{\mathrm{Dy}}$ refer to the prefactor $k_{s}$ obtained for the Dy/Sc SL and bulk Dy, respectively. For the $\mathrm{Ho}_{8} / \mathrm{Lu}_{18} \mathrm{SL}$ : (e) $m$ vs $t$, for bulk Ho (red line) and the Ho/Lu SL (black dots). The graph in the set shows the zero-field-cooled magnetization vs temperature for $\mu_{0} H=30 \mathrm{mT}$ applied along the $b$ axis. (e) $M-H$ loops, where $H$ is along the $b$ axis. See text for further details.

vector [41]. Experimental details on sample rotation and torque experiments can be found elsewhere [29,38,39,41]. The magnetic torque $L_{k}$ reads as $L_{k}=-M_{\perp}(\phi) H$ [29], where $H$ is the applied magnetic field, $M_{\perp}$ is the transversal component with respect to $H$ of the total magnetization $M$, and $\phi$ is the crystal angle, which is also obtained from the $\mathbf{M}$ in-plane components [39]. $L_{k}$ is typically investigated for a field range of $\mu_{0} H=1.5-2 \mathrm{~T}$, for which a forced ferromagnetic (FM) state is formed.

High-resolution $\mathrm{x}$-ray diffraction (XRD) experiments indicate that the Ho/Lu [36,37] and Dy/Sc [38] SLs are good single crystals. XRD data analysis shows that in the former, the coherent length $\lambda$ along the growth direction is about $\sim 150 \mathrm{~nm}$, the Ho layer develops an out-of-plane strain of $\sim 0.6 \%$, and the Ho layer thickness $n_{\mathrm{Ho}}$ is $\sim 8 \mathrm{MLs}$. In the latter, $\lambda$ turns out to be shorter, i.e., $\sim 59 \mathrm{~nm}$, the Dy layer out-of-plane strain is larger, which is $\sim 1.15 \%$, and the Dy layer thickness $n_{\text {Dy }}$ is also $\sim 8$ MLs. Complementarily, high-resolution transmission electron microscopy images of the Dy/Sc [38] SL showed that the Dy/Sc bilayer thickness is $\sim 4.4 \mathrm{~nm}$, the Dy-Sc interface roughness $\sigma$ amounts to $\pm 1 \mathrm{ML}$, and that $n_{\text {Dy }}$ is $\sim 8 \mathrm{ML}$ [see Fig. 1(a)], fully consistent with XRD data [38].

Neutron and magnetization studies in Dy/Sc [42] and Ho/Lu [37] SLs indicate that the magnetic moments are rigidly confined to the basal plane (BP) in the hcp lattice, as occurs in the bulk counterparts [30,31]. Furthermore, prior studies $[38,42]$ in Dy/Sc SL indicated that the magnetic coherent length $\lambda_{m} \approx n_{\text {Dy }}$, which means that adjacent Dy layers are magnetically decoupled through the Sc ones. Consistently, the magnetic response of the noninteracting Dy layers in the Dy/Sc SL is clearly reflected in the hysteresis loops, which exhibit a characteristic shape [38], resulting in a maximum ratio between remanence and saturation magnetization close to 0.5 , and in the variation of the coercive field $H_{c}$ with temperature, which follows a well-established relationship $H_{c}=H_{c}^{*}\left(1-t^{1 / 2}\right)$ [43], where $t=T / T_{C, N}$ is the reduced temperature and $T_{C, N}$ is the Curie or Neél ordering temperature, as appropriate [see Figs. 1(b) and 1(c)]. In contrast, in the ultrathin-alike Ho/Lu SL, $\lambda_{m}$ is $\sim 35 \mathrm{~nm}$ [37], spanning over at least five $\mathrm{Ho} / \mathrm{Lu}$ bilayers, which denotes that adjacent Ho layers are strongly coupled through the Lu ones. In RE/Sc SLs $[42,44]$ the interlayer exchange coupling appears to be singular. Thus, the absence of long-range coherence of the ordered Ho moments over the Sc layers in Ho/Sc SLs might be accounted for by considering the structural properties, the strain-induced modifications on the electronic band structure, and the influence of dipolar forces, as earlier noted [44].

As a result of the epitaxial strain [45], $T_{C}$ is shifted towards higher temperature in both SLs, which is $T_{C}=143 \mathrm{~K}$ in the Dy/Sc [38] SL and $T_{C}=35 \mathrm{~K}$ in the Ho/Lu SL [see Fig. 1(e)], when compared to $T_{C}=89 \mathrm{~K}$ [46] and $18 \mathrm{~K}$ [47] in Dy and Ho bulk, respectively. Additionally, the spin-spiral antiferromagnetic (AFM) phase is completely suppressed in the Dy/Sc [38], whereas that in the $\mathrm{Ho} / \mathrm{Lu} \mathrm{SL}$ is $T_{N}=115 \mathrm{~K}$, reaching a lower AFM ordering temperature than in bulk Ho [47] as a direct consequence of the finite size of the Ho layers [18].

Equally revealing is the temperature decay of the spontaneous reduced magnetization $m_{s}$ in the Dy/Sc SL. It is found that $m_{s}(T)$ is well modeled by a modified spin-wave theory [48] [see Fig. 1(d)], which predicts a decay of a surface, or two-dimensional-like, $M(T)$ according to a pseudo Bloch- $T^{3 / 2}$ power law with a prefactor $k_{s}$ which ranges from 3 to 5.4 [49]. The best-fit prefactor in the Dy/Sc SL is $k_{\mathrm{SL}}=3.5$, which produces an excellent accord between experiment and theory for $t \leqslant 0.5$. We note that in obtaining $k_{\mathrm{SL}}$ we took into account the energy gap [50] created in the magnon spectra by the MAE - the same applies to the case of bulk Dy. Importantly, the fact that $k_{\mathrm{SL}}=3.5$ indicates a notable softening of the indirect-exchange coupling transversal to the interfaces [49] in the Dy/Sc SL, as a result of the finite size of the noninteracting Dy layers. An essential feature of RE magnetism is a long spin-spin exchange coupling length $\xi$ which is at least of $\sim 40 \mathrm{MLs}$ [51]. Therefore, bearing in mind that $\xi \gg n_{\text {Dy }}=8 \mathrm{MLs}$, it is not surprising that the Dy layers in the Dy/Sc SL behave as a collection of noninteracting twodimensional ferromagnetic layers. This fact is fully consistent with a dimensional crossover transition from $3 \mathrm{D}$ to $2 \mathrm{D}$ in ultrathin RE layers, which is featured by a critical thickness of $15 \mathrm{MLs}$ [52]. In turn, the ultrathin- and strained-alike $\mathrm{Ho} / \mathrm{Lu} \mathrm{SL}$ behaves as a 3D magnetic nanostructure [37], forming a coherent FM phase below $T<T_{C}$, as suggested by the squareness of the $M-H$ loops, and the onset of a coherent spin-spiral phase for $T>T_{C}$, as pointed by the negligible remanence magnetization and the slope changes 

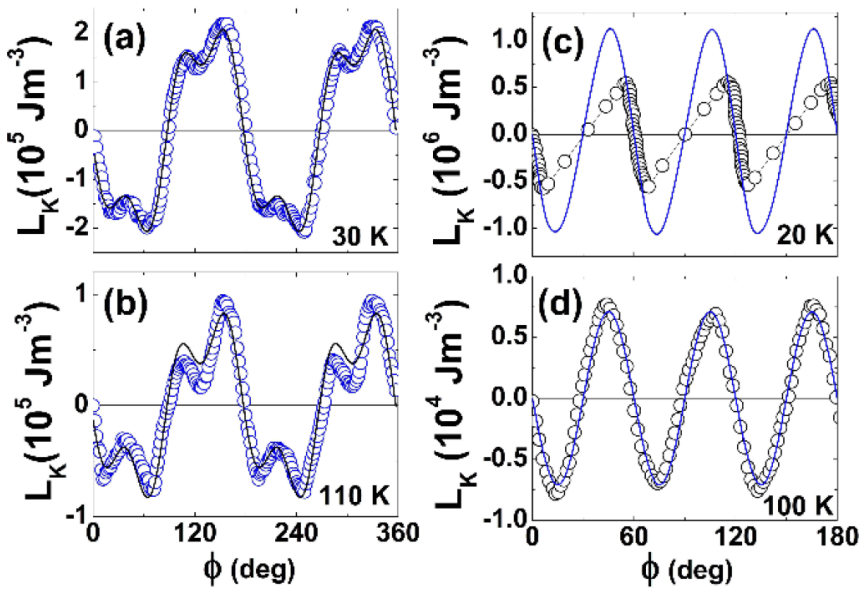

FIG. 2. (Color online) Magnetic torque $L_{k}$ as a function of the crystal angle $\phi$, where the applied magnetic field, $\mu_{0} H=2 \mathrm{~T}$, is in the c plane of the hcp lattice of the $\mathrm{Dy}_{8} / \mathrm{Sc}_{8} \mathrm{SL}$ (left-side panels) and $\mathrm{Ho}_{8} / \mathrm{Lu}_{18} \mathrm{SL}$ (right-side panels). The continuous lines are a fitting of the experimental data (circles) by utilizing the relationship $L_{k}=2 K_{2}^{\text {ef }} \sin 2 \phi+6 K_{6}^{\text {ef, } 6} \sin 6\left(\phi+\phi_{o}\right)$ [38], where $K_{2}^{\text {ef }}$ and $K_{6}^{\mathrm{ef}, 6}$ are the in-plane effective uniaxial and sixfold magnetic anisotropy constants, so that the best-fitting parameters are (a) $K_{2}^{\text {ef }}=$ $100 \mathrm{~kJ} \mathrm{~m}^{-3}$ and $K_{6}^{\mathrm{ef}, 6}=10.2 \mathrm{~kJ} \mathrm{~m}^{-3}$, and (b) $K_{2}^{\mathrm{ef}}=35.2 \mathrm{~kJ} \mathrm{~m}^{-3}$ and $K_{6}^{\text {ef, } 6}=4.9 \mathrm{~kJ} \mathrm{~m}^{-3}$, so that $\phi_{0}=5^{\circ}$ at both temperatures; (c) $K_{6}^{\text {ef, } 6}=0.2 \mathrm{MJ} \mathrm{m}^{-3}$ and (d) $K_{6}^{\text {ef, } 6}=1.18 \mathrm{~kJ} \mathrm{~m}^{-3}$, where $K_{2}^{\text {ef }}=0$ and $\phi_{0}$ is null in the case of the $\mathrm{Ho}_{8} / \mathrm{Lu}_{18} \mathrm{SL}$. For further details, see text.

(critical fields) manifested by the $M-H$ loop at $T=80 \mathrm{~K}$ [see Fig. 1(f)], which are typical of spin-spiral magnets [53]. Additionally, $m_{s}$ in the Ho/Lu SL varies with temperature slightly faster, albeit similar to that in bulk Ho [see Fig. 1(e)].

Due to the localized nature of the $4 f$ electrons [30] in lanthanides, in RE-based SLs the MAE is primarily modified by epitaxial strains $[29,38]$. We thereby opted to investigate strained-alike Dy/Sc and Ho/Lu SLs, wherein the Dy and Ho layers behave as 2D and 3D systems, respectively, because this particular setting enabled us to disentangle the distinctive way in which the finite size and the spin's low dimensionality [34] modify MAE, benefiting from the absence of thicknessdependent in- and out-of-plane spin reorientations (where the critical thickness is also temperature dependent), contrary to what occurs in $3 d$ ultrathin layers [54,55]. Additionally, the appearance of high-rank MAE constants-up to sixth order-in RE metals [30], alongside the emergence of lowsymmetry strain-induced MAE constants [38] in RE-based ultrathin layers, offers a unique testing ground in order to explore theoretical predictions [34].

At a glance, two main aspects stand out from inspecting the measured $L_{k}$ : First, it is visible that in the Dy/Sc SL [see Figs. 2(a) and 2(b)], $L_{k}$ shows the coexistence of twofold and sixfold symmetries, whereas in the Ho/Lu SL, $L_{k}$ only shows the characteristic sixfold symmetry manifested by RE metals [see Figs. 2(c) and 2(d)]; second, $L_{k}$ appears to decay faster with temperature in the $\mathrm{Ho} / \mathrm{Lu} \mathrm{SL}$ than in the Dy/Sc one. In both cases, $L_{k}$ is well fitted by employing the following relationship [38],

$$
L_{k}(\phi)=2 K_{2}^{\mathrm{ef}} \sin 2 \phi+6 K_{6}^{\mathrm{ef}, 6} \sin 6\left(\phi+\phi_{0}\right),
$$

where $K_{2}^{\text {ef }}$ and $K_{6}^{\text {ef, } 6}$ are the in-plane effective uniaxial and sixfold magnetic anisotropy constants, and $\phi_{0}$ is the shift angle between the uniaxial direction and the hexagonal lattice. For the Dy/Sc SL, the best fit is attained when $K_{2}^{\mathrm{ef}} \gg K_{6}^{\mathrm{ef}, 6}$ and $\phi_{0}=5^{\circ}$ [38], whereas the best fit in the Ho/Lu SL is achieved by inserting $K_{2}^{\text {ef }}=0\left(\phi_{0}=0\right)$ and $K_{6}^{\text {ef, } 6} \neq 0$ in Eq. (1) (see Fig. 2 for the resulting best-fit parameters). A recent study [38] has showed that $K_{2}^{\text {ef }}=-\varepsilon_{\gamma 1} M_{\gamma 2}^{2}$, where $M_{\gamma 2}^{2}$ is a MEL constant associated with the $\gamma$ strictions in hexagonal symmetry [56], so that $M_{\gamma_{2}}^{2} \sim 1 \mathrm{GPa}$ in the Dy/Sc SL, and $\varepsilon_{\gamma 1} \sim 10^{-4}$ is the epitaxial orthorhombiclike strain, whose origin resides in the in-plane anisotropic strain relaxation process between the buffer and seed bottom layers [38] in the superstructure. Considering that $\varepsilon_{\gamma_{1}}$ may equally emerge in the Ho/Lu SL, and assuming this attains a similar order of magnitude, we ascribe the infeasibility of determining $K_{2}^{\text {ef }}$ in the Ho/Lu SL to the arising of a smaller symmetry-breaking MEL constant, i.e., $M_{\gamma 2}^{2} \leqslant 0.1 \mathrm{GPa}$ [57], when compared to $M_{\gamma 2}^{2}$ in the Dy/Sc SL.

Here, $L_{k}$ shows a shearlike dependence on $\phi$ in the $\mathrm{Ho} / \mathrm{Lu} \mathrm{SL}$ at $T=20 \mathrm{~K}$, given that the anisotropy field is large compared to $H$ [41] [see Fig. 2(c)]. In this case, the field-independent $K_{6}^{\text {ef, } 6}$ is obtained employing a graphical method, so that $K_{6}^{\text {ef, } 6}$ in Eq. (1) is chosen to match the slope of $L_{k}$ around the easy axis. For higher temperatures, the field-independent MAE constants have been obtained following well-established methods [38]. The temperature dependence of the MAE constants is not only a fingerprint of its microscopic origin [25,29], but also is markedly reflected in the spin's dimensionality [34]. Overall, the CC theory [25] predicts a notably faster decay for $K_{2}^{\mathrm{ef}}$ and $K_{6}^{\mathrm{ef}, 6}$ in the Dy/Sc SL than that experimentally observed. This discrepancy is particularly striking for $K_{6}^{\mathrm{ef}, 6}$, but less pronounced in the case of $K_{2}^{\mathrm{ef}}$; even so, notice that $K_{2}^{\text {ef }}$ is underestimated by as much as $20 \%-25 \%$ when compared to the CC model [see Fig. 3(a)]. By contrast, the CC theory [25] models very well $K_{6}^{6, \text { ef }}$ in the Ho/Lu SL, provided magnetostrictive [29] and strain-induced [29,38] MEL terms are considered, so that the best-fit parameters are $K_{6, m c}^{6, m e}=-0.3 \mathrm{MJ} \mathrm{m}^{-3}$ and $K_{6}^{6, m e}=0.55 \mathrm{MJ} \mathrm{m}^{-3}$ [see Fig. 3(b)]. We notice that these values do not fit with an earlier study [29] of the MAE in a set of Ho/Lu SLs. To account for that, we should consider that in RE-based SLs, the interface magnetic anisotropy term appears to be very small [29]. This is so because the symmetry of the crystal lattice is preserved across the Ho-Lu interfaces and the redistribution of the conduction electron density that might take place at and across the interfaces, resulting from the electronic band mismatch, would originate a small change in the screening of the localized $4 f$ electron densities, resulting in a second-order modification of the magnetocrystalline anisotropy. In addition, the mixing at the interfaces, visualized in the $\mathrm{Ho} / \mathrm{Lu}$ system as the forming of an 2.5 ML thick Ho-Lu alloy layer [36], is expected to negligibly modify the crystal field [58] acting on the $4 f$ moments, leading to that the magnetocrystalline anisotropy of the Ho atomic layers is homogeneous across the layer. We therefore assign the reduced value obtained for $K_{6}^{6, m e}$, compared to that measured across the previous $\mathrm{Ho} / \mathrm{Lu}$ series [29], to be the result of the appearance of an $\varepsilon_{\gamma_{1}}$. If this 

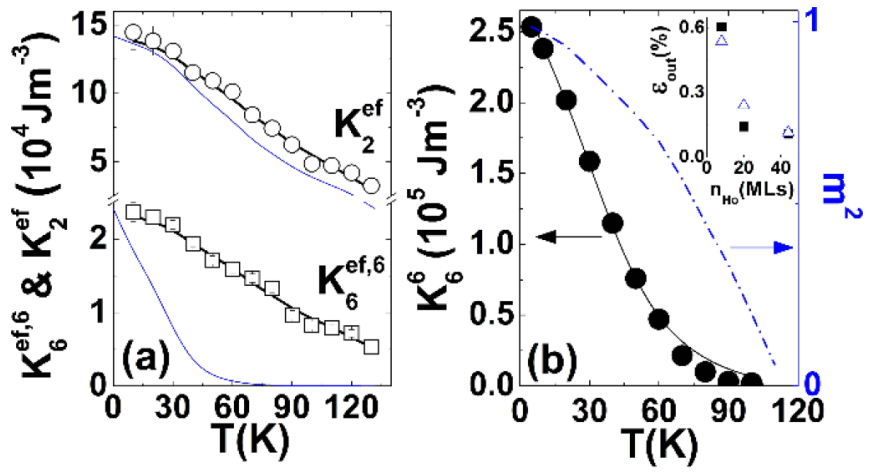

FIG. 3. (Color online) (a) Temperature dependence of the uniaxial (circles), $K_{2}^{\text {ef }}$, and hexagonal (squares), $K_{6}^{\text {ef, } 6}$, magnetic anisotropy constants for the Dy/Sc SL. The black lines are a fit according to the relationship $K=K(0) M^{2}$ [34], where $m=M(T) / M(0)$ and $M$ is the total magnetization, so that the best-fit parameters are $K_{2}^{\mathrm{ef}}(0)=226 \mathrm{~kJ} \mathrm{~m}^{-3}$ and $K_{6}^{\mathrm{ef}, 6}(0)=12.8 \mathrm{~kJ} \mathrm{~m}^{-3}$. The blue lines correspond to a fitting function according to the single-ion model [25], which is $K_{2}^{\text {ef }}=K_{2}^{\text {ef }}(0) \widehat{I}_{5 / 2}[\widehat{m}]$ and $K_{6}^{\text {ef, } 6}=K_{2}^{\text {ef }}(0) \widehat{I}_{13 / 2}[\widehat{m}]$, where $\widehat{I}_{(2 l+1) / 2}[\widehat{m}]$ for $l=2,6$ are the normalized hyperbolic Bessel functions and $\widehat{m} \equiv \mathcal{L}^{-1}[m(T)]$, where $\mathcal{L}^{-1}$ is the inverse Langevin function. (b) Temperature variation of $K_{6}^{\mathrm{ef}, 6}$ (circles) and $m^{2}$ (dashed-dotted line) for the $\left[\mathrm{Ho}_{8} / \mathrm{Lu}_{18}\right]_{75} \mathrm{SL}$. The continuous line is a fit according to the relationship $K_{6}^{\text {ef, } 6}=K_{6, m c}^{6, m e} \widehat{I}_{13 / 2}[\hat{m}]+K_{6}^{6, m e} \widehat{I}_{5 / 2}[\hat{m}] \widehat{I}_{9 / 2}[\hat{m}]$ [29], so that the best-fit parameters are $K_{6, m c}^{6, m e}(0)=-0.3 \mathrm{MJ} \mathrm{m}^{-3}$ and $K_{6}^{6, m e}(0)=0.55 \mathrm{MJ} \mathrm{m}^{-3}$. The graph in the inset displays the measured [37] (squares) out-of-plane strain $\varepsilon_{\text {out }}$ and the estimated one (triangles) according to an elastic model [29] tested in a set of $\mathrm{Ho} / \mathrm{Lu}$ superlattices vs the Ho layer thickness $n_{\mathrm{Ho}}$.

is the case, the $\gamma$-striction-related MEL terms $-M_{\gamma 2}^{2} M_{\gamma 2}^{4} / c_{\gamma}$ and $-M_{\gamma 2}^{4} M_{\gamma 2}^{4} / c_{\gamma}$, which contribute to $K_{6}^{6, m e}$ and $K_{6, m c}^{6, m e}$ [56], respectively, will become strain dependent, where $c_{\gamma}$ is the symmetric elastic constant. Thus, assuming a linear approximation for small strains [38], we obtain that $M_{\gamma 2(4)}^{2(4)}\left(\varepsilon_{\gamma 1}\right) \simeq$ $M_{\gamma 2(4)}^{2(4)}(0)+N_{\gamma 2(4)}^{2(4)} \varepsilon_{\gamma 1}$, where $N_{\gamma 2(4)}^{2(4)}=\partial M_{\gamma 2(4)}^{2(4)} /\left.\partial \varepsilon_{\gamma 1}\right|_{\varepsilon_{\gamma 1}=0}$, so that terms such as $-\left(M_{\gamma 2}^{2(4)} N_{\gamma 4}^{4}+M_{\gamma 4}^{4} N_{\gamma 2}^{2(4)}\right) \varepsilon_{\gamma 1} / c_{\gamma}$ will now contribute to $K_{6}^{6, m e}\left(K_{6, m c}^{6, m e}\right)$, posing an almost indistinguishable temperature variation as to those unstrained terms [56], provided $\varepsilon_{\gamma 1}$ slowly varies with temperature, such as occurs [38]. The strain state of the ultrathin Ho layers is complex to be determined with high precision [38], turning highly anisotropic [see the inset in Fig. 3(b)], and this might favor the appearance of $\varepsilon_{\gamma 1}$, as occurs in Dy/Sc SLs [38] and contrary to Ho/Lu SLs with a larger $n_{\text {Ho }}$ [29].

An extension of the CC theory [34] to ultrathin magnetic layers predicts that, in the case of 2D magnets, a generic MAE constant $K_{n}^{l}$ scales with temperature as follows:

$$
K_{n}^{l} \approx K_{n}^{l}(0) m^{\alpha}(T),
$$

where $K_{n}^{l}(0)$ is the extrapolated value at $T=0 \mathrm{~K}, m=$ $M(T) / M(0)$ and $M$ is the magnetization of the $2 \mathrm{D}$ spin system, and the exponent takes the values $\alpha=l^{2}$ at very low temperatures, which is for $m \simeq 1$, and $\alpha=2$ for high temperatures, i.e., for $m<1$. Despite the fact that theory does not predict a unique dependence on $m$, we however observe that a fitting function according to Eq. (2) with $\alpha=2$ produces an excellent agreement between the experiment and theory, so that the best-fit parameters are $K_{2}^{\mathrm{ef}}(0)=226 \mathrm{~kJ} \mathrm{~m}^{-3}$ and $K_{6}^{\text {ef, } 6}(0)=12.8 \mathrm{~kJ} \mathrm{~m}^{-3}$.

Here, $3 d$ metal ultrathin layers also develop high-rank MAE constants [55,59], which significantly contribute to spin reorientations. Huge efforts were devoted to explore the MAE of ultrathin layers; however, clear evidence regarding the universal temperature scaling of the MAE constants in 2D magnetic layers $[55,59,60]$ was not conclusively obtained upon until now. Thus, the most compelling studies focused on layers thicker than $5 \mathrm{ML}$, which behave as 3D nanostructures [61], whereas those studies performed on thinner layers centered on testing $K_{2}$ and utilized a model-dependent approach and researched a narrow temperature range.

In ultrathin films, the diminishing of the MAE with temperature is driven by magnon excitations [62]. Thus, SW dynamics in multilayers [63] notably differ from bulk metals, and this divergence grows wider in the case of ultrathin films [64]. Studies on Dy-based SLs [65] have shown that, if the Dy layers are decoupled, the SW energy dispersion relation turns discontinuous with discrete excitations. In the case of the Dy/Sc SL, the SW excitations will experience the quantization of the wave-vector transversal to the film plane, $q_{z}$, which will eventually lead to the appearance of a gap in the SW energy spectrum [66] with a minimum wave vector $q_{z}^{\min } \approx 0.13$, given in units of $2 d_{\mathrm{Dy}} / 2 \pi$, where $d_{\text {Dy }}=2.846 \AA[38]$ is the interatomic spacing along the hexagonal axis. Thus, the thermally driven SW excitations propagating along the $c$ axis for temperatures up to $T_{C}=$ $143 \mathrm{~K}$, which correspond to an energy of $\hbar \omega_{\mathrm{SW}} \approx K_{B} T<$ $1.3 \mathrm{meV}$, where $\omega_{\mathrm{S}}$ is the SW frequency, will be completely inaccessible [65]. The enhanced thermal stability of the spins in 2D magnets stems from the quantum confinement of the spin's fluttering driven by thermally induced quasi-2D magnons.

In conclusion, we have shown that the magnetic anisotropy in 2D ferromagnets scales with temperature as a universal quadratic power law of the reduced magnetization, in agreement with predictions, albeit in marked contrast to the rank-dependent, thereby faster, temperature decay of the MAE constants predicted by the single-ion theory in $3 \mathrm{D}$ nanostructures.

One of us (L.B.) acknowledges support from the 7th Frame Program of the European Commission (MSC Actions, Contract No. 025693 MEIF-CT-2006).
[1] C. Chappert, A. Fert, and F. Nguyen Van Dau, Nat. Mater. 6, 813 (2007); A. Fert, Rev. Mod. Phys. 80, 1517 (2008).

[2] D. Houssameddine et al., Nat. Mater. 6, 447 (2007).
[3] D. Suess, Appl. Phys. Lett. 89, 113105 (2006).

[4] A. V. Khvalkovskiy et al., J. Phys. D: Appl. Phys. 46, 074001 (2013). 
[5] S. Chikazumi, Physics of Magnetism (Wiley, New York, 1978).

[6] D. P. Pappas, K.-P. Kämper, and H. Hopster, Phys. Rev. Lett. 64, 3179 (1990).

[7] A. Hubert and R. Schäfer, Magnetic Domains (Springer, Berlin, 1998).

[8] G. Tatara and H. Kohno, Phys. Rev. Lett. 92, 086601 (2004).

[9] M. T. Johnson, P. J. H. Bloemen, F. J. A. den Broeder, and J. J. de Vries, Rep. Prog. Phys. 59, 1409 (1996).

[10] D. Sander, Rep. Prog. Phys. 62, 809 (1999); J. Phys.: Condens. Matter 16, R603 (2004).

[11] M. Albrecht, G. Hu, I. L. Guhr, T. C. Ulbrich, J. Boneberg, P. Leiderer, and G. Schatz, Nat. Mater. 4, 203 (2005); M. Weisheit, S. Fähler, A. Marty, Y. Souche, C. Poinsignon, and D. Givord, Science 315, 349 (2007).

[12] F. J. A. den Broeder, D. Kuiper, A. P. van de Mosselaer, and W. Hoving, Phys. Rev. Lett. 60, 2769 (1988); M. Jamet, W. Wernsdorfer, C. Thirion, D. Mailly, V. Dupuis, P. Mélinon, and A. Pérez, ibid. 86, 4676 (2001).

[13] P. Gambardella, A. Dallmeyer, K. Maiti, M. C. Malagoli, W. Eberhardt, K. Kern, and C. Carbone, Nature (London) 416, 301 (2002)

[14] P. Gambardella et al., Science 300, 1130 (2003).

[15] S. Rusponi, T. Cren, N. Weiss, M. Epple, P. Buluschek, L. Claide, and H. Brune, Nat. Mater. 2, 546 (2003).

[16] C. M. Schneider, P. Bressler, P. Schuster, J. Kirschner, J. J. de Miguel, and R. Miranda, Phys. Rev. Lett. 64, 1059 (1990).

[17] R. Zhang and R. F. Willis, Phys. Rev. Lett. 86, 2665 (2001).

[18] E. Weschke et al., Phys. Rev. Lett. 93, 157204 (2004).

[19] U. Gradmann and J. Müller, Phys. Status Solidi 27, 313 (1968).

[20] S. Blundell, Magnetism in Condensed Matter (Oxford University Press, Oxford, UK, 2001).

[21] N. D. Mermin and H. Wagner, Phys. Rev. Lett. 17, 1133 (1966).

[22] A. Brinkman, M. Huijben, M. van Zalk, J. Huijben, U. Zeitler, J. C. Maan, W. G. van der Wiel, G. Rijnders, D. H. A. Blank, and H. Hilgenkamp, Nat. Mater. 6, 493 (2007); H. X. Yang, A. Hallal, D. Terrade, X. Waintal, S. Roche, and M. Chshiev, Phys. Rev. Lett. 110, 046603 (2013); Y. Ma, Y. Dai, M. Guo, C. Niu, Y. Zhu, and B. Huang, ACS Nano 6, 1695 (2012); S. Farokhipoor et al., Nature (London) 515, 379 (2014).

[23] A. K. Geim, Science 324, 1530 (2009); Q. H. Wang, K. Kalantar-Zadeh, A. Kis, J. N. Coleman, and M. S. Strano, Nat. Nanotechnol. 7, 699 (2012).

[24] P. Krams, F. Lauks, R. L. Stamps, B. Hillebrands, and G. Güntherodt, Phys. Rev. Lett. 69, 3674 (1992).

[25] E. R. Callen and H. B. Callen, J. Phys. Chem. Solids 27, 1271 (1966).

[26] U. Welp, A. Berger, V. K. Vlasko-Vlasov, Q. A. Li, K. E. Gray, and J. F. Mitchell, Phys. Rev. B 62, 8615 (2000).

[27] X. W. Wu, K. Y. Guslienko, R. W. Chantrell, and D. Weller, Appl. Phys. Lett. 82, 3475 (2003).

[28] K.-Y. Wang, M. Sawicki, K. W. Edmonds, R. P. Campion, S. Maat, C. T. Foxon, B. L. Gallagher, and T. Dietl, Phys. Rev. Lett. 95, 217204 (2005).

[29] L. Benito, J. I. Arnaudas, M. Ciria, C. de la Fuente, A. del Moral, R. C. C. Ward, and M. R. Wells, Phys. Rev. B 70, 052403 (2004).

[30] Magnetic Properties of Rare Earth Metals, edited by R. J. Elliot (Plenum, London, 1972).

[31] J. J. Rhyne and A. E. Clark, J. Appl. Phys. 38, 1379 (1967).
[32] M. D. Kuz'min, Phys. Rev. B 46, 8219 (1992); F. Herbst, Rev. Mod. Phys. 63, 819 (1991); N. C. Koon, C. M. Williams, and B. N. Das, J. Magn. Magn. Mater. 100, 173 (1991).

[33] C. F. Majkrzak, J. Kwo, M. Hong, Y. Yafet, D. Gibbs, C. L. Chien, and J. Bohr, Adv. Phys. 40, 99 (1991); J. J. Rhyne and R. W. Erwin, in Handbook of Magnetic Materials, edited by K. H. Buschow (North-Holland, Amsterdam, 1995), Vol. 8.

[34] E. Callen, J. Appl. Phys. 53, 8139 (1982).

[35] R. C. C. Ward, M. R. Wells, C. Bryn-Jacobsen, R. A. Cowley, D. F. McMorrow, and J. A. Simpson, Thin Solid Films 275, 137 (1996).

[36] D. F. McMorrow, P. P. Swaddling, R. A. Cowley, R. C. C. Ward, and M. R. Wells, J. Phys.: Condens. Matter 8, 6553 (1996).

[37] P. P. Swaddling, R. A. Cowley, R. C. C. Ward, M. R. Wells, and D. F. McMorrow, Phys. Rev. B 53, 6488 (1996).

[38] L. Benito, C. Ballesteros, and R. C. C. Ward, Phys. Rev. B 89 , $134421(2014)$

[39] L. Benito, J. I. Arnaudas, and A. del Moral, Rev. Sci. Instrum. 77, 025101 (2006).

[40] L. Benito, M. Ciria, C. de la Fuente, J. I. Arnaudas, R. C. C Ward, and M. R. Wells, Phys. Rev. Lett. 94, 227204 (2005); L. Benito, M. Ciria, A. Fraile, D. Fort, J. S. Abell, and J. I. Arnaudas, ibid. 98, 267201 (2007).

[41] L. Benito, K. Dumesnil, and R. C. C. Ward, Phys. Rev. B 90, 054407 (2014).

[42] F. Tsui, C. P. Flynn, R. S. Beach, J. A. Borchers, R. W. Erwin, and J. J. Rhyne, J. Appl. Phys. 73, 6904 (1993); F. Tsui, C. Uher, and C. P. Flynn, Phys. Rev. Lett. 72, 3084 (1994).

[43] E. C. Stoner and E. P. Wohlfarth, Philos. Trans. R. Soc. London, Ser. A 240, 599 (1948).

[44] C. Bryn-Jacobsen, R. A. Cowley, D. F. McMorrow, J. P. Goff, R. C. C. Ward, and M. R. Wells, Phys. Rev. B 55, 317 (1997).

[45] R. S. Beach, J. A. Borchers, A. Matheny, R. W. Erwin, M. B. Salamon, B. Everitt, K. Pettit, J. J. Rhyne, and C. P. Flynn, Phys. Rev. Lett. 70, 3502 (1993).

[46] M. K. Wilkinson, W. C. Koehler, E. O. Wallan, and J. W. Cable, J. Appl. Phys. 32, S48 (1961).

[47] W. C. Koehler, J. W. Cable, M. K. Wilkinson, and E. O. Wollan, Phys. Rev. 151, 414 (1966).

[48] J. Mathon and S. B. Ahmad, Phys. Rev. B 37, 660 (1988).

[49] D. Mauri, D. Scholl, H. C. Siegmann, and E. Kay, Phys. Rev. Lett. 61, 758 (1988); B. S. Ahmad, J. Mathon, and M. S. Phan, J. Phys., Colloq. (Paris) 49, C8-1639 (1988).

[50] A. R. Mackintosh, Phys. Lett. 4, 140 (1963).

[51] J. J. Rhyne, R. W. Erwin, J. Borchers, S. Sinha, M. B. Salamon, R. Du, and C. P. Flynn, J. Appl. Phys. 61, 4043 (1987); D. A. Jehan, D. F. McMorrow, R. A. Cowley, R. C. C. Ward, M. R. Wells, N. Hagmann, and K. N. Clausen, Phys. Rev. B 48, 5594 (1993).

[52] A. Aspelmeier, F. Gerhardter, and K. Baberschke, J. Magn. Magn. Mater. 132, 22 (1994).

[53] L. Benito, R. C. C. Ward, and M. G. Blamire, Phys. Rev. B 88 , 224407 (2013).

[54] B. Schulz and K. Baberschke, Phys. Rev. B 50, 13467 (1994), and references therein; M. Farle, W. Platow, A. N. Anisimov, P. Poulopoulos, and K. Baberschke, ibid. 56, 5100 (1997).

[55] K. Baberschke and M. Farle, J. Appl. Phys. 81, 5038 (1997).

[56] L. Benito, J. I. Arnaudas, M. Ciria, C. de la Fuente, and A. del Moral, J. Phys.: Condens. Matter 16, 7151 (2004). 
[57] A. del Moral, M. Ciria, J. I. Arnaudas, M. R. Wells, R. C. C. Ward, and C. de la Fuente, J. Phys.: Condens. Matter 10, L139 (1998).

[58] P. Touborg and J. Høg, Phys. Rev. Lett. 33, 775 (1974); P. Touborg, Phys. Rev. B 16, 1201 (1977).

[59] M. Farle, B. Mirwald-Schulz, A. N. Anisimov, W. Platow, and K. Baberschke, Phys. Rev. B 55, 3708 (1997); M. Farle, A. N. Anisimov, W. Platow, P. Poulopoulos, and K. Baberschke, J. Magn. Magn. Mater. 198-199, 325 (1999).

[60] D. P. Pappas, J. Vac. Sci. Technol. B 14, 3203 (1996); M. Farle, W. Platow, A. N. Anisimov, B. Schulz, and K. Baberschke, J. Magn. Magn. Mater. 165, 74 (1997); Kh. Zakeri, Th. Kebe, J. Lindner, and M. Farle, Phys. Rev. B 73, 052405 (2006); B. Aktaş, B. Heinrich, G. Woltersdorf, R. Urban, L. R. Tagirov,
F. Yildiz, K. Özdogan, M. Özdemir, O. Yalçin, and B. Z. Rameev, J. Appl. Phys. 102, 013912 (2007).

[61] Y. Li and K. Baberschke, Phys. Rev. Lett. 68, 1208 (1992).

[62] J. Kienert, S. Schwieger, K. Lenz, J. Lindner, K. Baberschke, and W. Nolting, J. Magn. Magn. Mater. 316, e86 (2007).

[63] A. T. D. Grünwald, A. R. Wildes, W. Schmidt, E. V. Tartakovskaya, J. Kwo, C. Majkrzak, R. C. C. Ward, and A. Schreyer, Phys. Rev. B 82, 014426 (2010).

[64] J. Prokop, W. X. Tang, Y. Zhang, I. Tudosa, T. R. F. Peixoto, Kh. Zakeri, and J. Kirschner, Phys. Rev. Lett. 102, 177206 (2009).

[65] J. T. Haraldsen and R. S. Fishman, J. Phys.: Condens. Matter 22, 186002 (2010).

[66] V. Ya. Demikhovskii and B. A. Tavger, JETP 18, 797 (1964). 